\title{
Assessment of function of contractile segments in patients with left ventricular aneurysms by quantitative first pass radionuclide ventriculography Haemodynamic correlation at rest and exercise ${ }^{\star}$
}

\author{
DUNCAN S DYMOND, JOHN STEPHENS, DAVID STONE, P H JARRITT, \\ ALEX ELLIOTT, K E BRITTON, R A J SPURRELL
}

From the Departments of Cardiology and Nuclear Medicine, St. Bartholomew's Hospital, London

SUMMARY Thirteen patients with left ventricular aneurysms complicating myocardial infarction were studied by contrast angiography and by first pass radionuclide ventriculography. The ejection fraction of the contractile segment (EFCS) was measured from both studies using a double hemispheroid model, and the values correlated closely. There was a monotonic relation between EFCS and stroke volume index measured from thermodilution cardiac outputs carried out simultaneously with the radionuclide study When radionuclide ventriculography was performed at submaximal supine exercise, changes in EFCS paralleled changes in the total left ventricular ejection fraction in 10 of the 13 cases.

In nine patients, changes in EFCS paralleled changes in stroke volume index and the relation between EFCS and stroke volume index was maintained at exercise. After administration of the vasodilator isosorbide dinitrate to 12 patients, repeat exercise radionuclide ventriculography showed an improvement in left ventricular ejection fraction and in eight patients EFCS improved.

First pass radionuclide ventriculography can accurately estimate EFCS, which may be an important factor in predicting the likely response to aneurysmectomy. Changes in EFCS on exercise are reflected in changes in total left ventricular ejection fraction and stroke volume index. Isosorbide dinitrate may improve contractile segment function on exercise.

Surgical resection of left ventricular aneurysms complicating myocardial infarction is a wellestablished therapeutic technique, though there are wide ranges in both the reported in-hospital mortality and the long-term mortality, ${ }^{1-5}$ and the procedure is by no means certain to produce clinical improvement. The crucial factors in the assessment of patients being considered for aneurysmectomy are the demonstration of segmental rather than diffuse ventricular dysfunction and the presence of sufficiently good function of the contractile portion of ventricle. The differentiation between segmental and diffuse ventricular disease has for many years been accomplished by contrast left

* This work was supported by the British Heart Foundation.

† Present address: Department of Nuclear Medicine, Middlesex Hospital, London W1N 8AA.

Received for publication 19 June 1979 ventriculography, ${ }^{6}$ but contractile segment function has often been assessed by visual inspection of the cineangiogram, rather than by objective quantification. Recently, attempts have been made to quantify contractile segment function angiographically and to relate this function to the risks and results of aneurysmectomy. ${ }^{78}$ These studies have suggested that a contractile segment ejection fraction of approximately 40 per cent is necessary for a good result from aneurysmectomy.

The ability of radionuclide ventriculography to detect left ventricular aneurysms and to discriminate between aneurysms and diffuse hypokinesis is well documented. ${ }^{9} 10$ This study describes the quantification of contractile segment function by radionuclide ventriculography and haemodynamic correlates of this function at rest and on exercise. The effect of isosorbide dinitrate on left ventricular function on exercise is also assessed. 


\section{Patients and methods}

Thirteen patients with left ventricular aneurysms occurring after myocardial infarction were studied. There were 11 men and two women. Their ages ranged from 44 to 64 years with a mean age of 56 years. The main reason for investigation was cardiac failure in 10, angina pectoris in two, and recurrent ventricular arrhythmias in one. Informed consent was obtained in all cases. Left heart catheterisation was performed via a right brachial arteriotomy, left ventriculography being carried out in the right anterior oblique projection. A flow-directed thermodilution catheter was positioned in the pulmonary artery. In eight patients the haemodynamic measurements were made at the time of catheterisation with the following procedure. Thirty minutes were allowed to lapse for the effects of contrast media to subside $^{11}$ and control measurements were made of cardiac output by the thermodilution technique, ${ }^{12}$ using a Waters cardiac output computer (COR $100 \mathrm{~A}$ ), and of heart rate. All measurements were made in triplicate. The patients then performed submaximal supine leg exercise with a bicycle ergometer, at a previously determined work rate that caused a rise in heart rate of between 30 and 50 per cent over resting levels, but that did not cause limiting dyspnoea or angina pectoris. Measurements were then repeated. Submaximal exercise was employed in these patients as all were severely symptomatic. They would have been unable to maintain exercise for long enough to allow three sets of haemodynamic measurements to be made had higher workloads been used. After a 10 minute rest period, $5 \mathrm{mg}$ isosorbide dinitrate were administered sublingually. Fifteen minutes after the drug, by which time resting left ventricular filling pressure had fallen to $16 \mathrm{mmHg}$ or less in all patients, exercise was reperformed to the same level as previously and measurements repeated.

The other five patients were not exercised in the catheter laboratory but had all haemodynamic measurements performed at the time of radionuclide ventriculography.

A resting radionuclide ventriculogram was carried out in the right anterior oblique projection, within six hours of cardiac catheterisation in 10 patients and within 24 hours in the other three, using a computerised multicrystal gamma camera with high count-rate capabilities ${ }^{13}$ (Baird-Atomic, System 77). A $10 \mathrm{mCi}$ bolus of high specificactivity technetium ${ }^{90 \mathrm{~m}}$, as pertechnetate, was injected through the right atrial lumen of the thermodilution catheter with a rapid saline flush. One thousand frames of data were collected at $50 \mathrm{~ms}$ framing intervals to monitor the first pass of the radionuclide through the central circulation. A $1 \frac{1}{2}$ in parallel-hole collimator was used. Data were corrected for field non-uniformity and the dead time of the instrument, and stored on magnetic tape. The day after the resting study, radionuclide ventriculography was performed at exercise, after an identical workload in the case of the eight patients who had exercised in the catheter laboratory. The other five patients were exercised using an identical procedure, haemodynamic measurements being made immediately before the cessation of exercise and the injection of the radionuclide. In 12 of the 13 patients, a further exercise radionuclide ventriculogram was performed 15 minutes after $5 \mathrm{mg}$ isosorbide dinitrate were administered sublingually, allowing at least 25 minutes between the two exercise studies. This period was allowed for the clearance of the majority of the first bolus of technetium $^{99 \mathrm{~m}}$ from the intracardiac blood pool. Before the second exercise study a static background frame was counted to a maximum of 9000 counts per cell and the second study corrected for residual blood pool activity as well as for field non-uniformity and dead time. Since constant patient positioning was crucial to enable accurate background blood pool correction, the positions of four light beams emitted by the detector head were marked on the patients' chests and adjusted as necessary before the injection of the post-isosorbide dinitrate radionuclide bolus.

There was no morbidity in any case and the maximum accumulated dose of technetium ${ }^{99} \mathrm{~m}$ for each patient was $35 \mathrm{mCi}$.

For this study, a ventricular aneurysm was defined angiographically as a clearly demarcated akinetic segment with or without associated areas of dyskinesis, ${ }^{14}$ separated from a contractile segment of ventricle, in sinus rhythm. Coronary artery stenoses of 70 per cent or more of the arterial lumen were judged to be significant.

For objective evaluation of the contrast angiograms end-diastolic and end-systolic frames were traced by selecting the largest and subsequent smallest appearing ventricular outlines of a beat that was not an ectopic, or immediately after an ectopic. Analysis of the radionuclide studies was carried out using software supplied by the manufacturers. Total left ventricular ejection fraction was calculated from the change in counts from enddiastole to end-systole, read from the high frequency background corrected time-activity curve from the left ventricular region of interest, ${ }^{10} 15$ making no assumptions about ventricular geometry. A representative cardiac cycle was generated by summing corresponding frames from the peaks and troughs of the time-activity curve. The perimeters of the 
ventricle at end-diastole and end-systole were generated after linear interpolation of the enddiastolic and end-systolic frames from a data matrix of 294 points $(14 \times 21)$ to one of 4704 points $(56 \times$ 84).

The computerised edge enhancement technique used for perimeter generation consisted of highlighting the 5 per cent count band corresponding to 25 to 30 per cent of the maximum end-diastolic count for both end-diastole and end-systole. This method of edge enhancement has been found to produce the most consistent agreement with contrast angiographic volumes. ${ }^{10}$

EFCS per cent was measured using the double hemispheroid model of Watson et al. ${ }^{8}$ In this model a line of demarcation is drawn between the section of ventricle that shows definite inward motion during systole, that is the contractile segment, and the akinetic or dyskinetic segment. EFCS was calculated from an area-length method modified to fit the double hemispheroid model, with the long axis of the segment being the longest length perpendicular to the line of demarcation. The areas and lengths were calculated from the traced perimeters of both contrast and radionuclide studies, using an electronic pressure pad linked to a computer, and an algorithm analogous to planimetry. For each area and length, three measurements were taken and meaned.

Stroke volume index was calculated from the mean of the thermodilution cardiac outputs, heart rate, and body surface area. Results were analysed by Student's t test and Spearman's rank test.

\section{Results}

Aneurysms were anterolateral or apical in 12 , one patient having a discrete inferior aneurysm. No

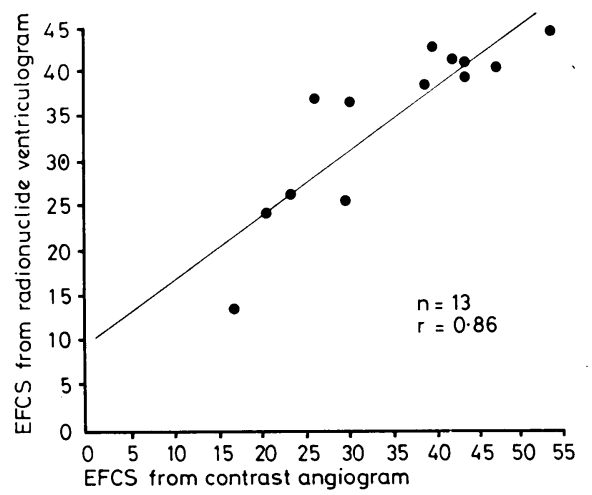

Fig. 1 Plot of EFCS from contrast angiogram against EFCS from radionuclide study. EFCS, ejection fraction of contractile segment.

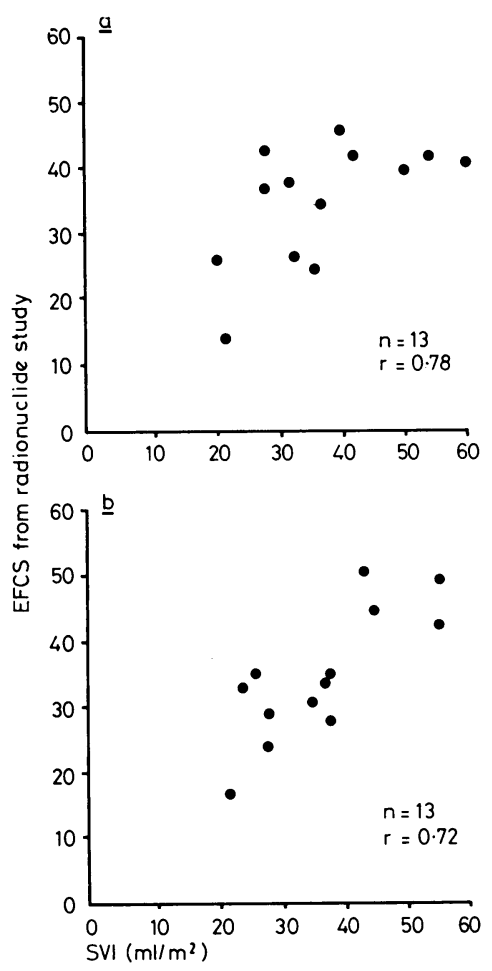

Fig. 2 (a) Correlation between stroke volume index (SVI) and EFCS at rest. (b) Correlation between stroke volume index (SVI) and EFCS at exercise. EFCS, ejection fraction of contractile segment.

patient had significant mitral regurgitation. Eight patients had one-vessel coronary disease, seven of these having an occluded left anterior descending artery, one an occluded right coronary. One had two-vessel disease and four had three-vessel disease.

EFCS from the contrast angiogram ranged from 17 to 53 per cent with a mean of 34.7 per cent, while EFCS from the radionuclide study at rest ranged from 14 to 46 per cent with a mean of 35.0 per cent. Total left ventricular ejection fraction was reduced in all patients, ranging from 19 to 37 per cent, with a mean of 27.0 per cent. The correlation between EFCS from contrast and radionuclide studies is shown in Fig. 1, the correlation coefficient being $0.86(p<0.001)$. There was a poor correlation between EFCS and total left ventricular ejection fraction $(r=0 \cdot 3)$. Thus total left ventricular ejection fraction could not be used to differentiate patients with good and bad contractile segment function. Stroke volume index ranged from 20 to $59 \mathrm{ml} / \mathrm{m}^{2}$, with a mean of $36 \mathrm{ml} / \mathrm{m}^{2}$. Fig. 2a shows the relation between resting EFCS and resting stroke volume index to be monotonic. Analysis of this relation by 
Spearman's non-parametric rank test shows a significant correlation with a coefficient of 0.78 . This is not surprising as by definition the contractile segment is the only ventricular segment contributing to cardiac. output.

Six patients had a resting EFCS of 40 per cent or more. Residual standard error from regression was used to estimate the predicted value of EFCS from the radionuclide angiogram which corresponded to a value of 40 per cent from the contrast angiogram. Thus EFCS (radionuclide) $=39 \cdot 2 \pm 3.0$ per cent at EFCS (contrast) of 40 per cent.

\section{EFFECTS OF EXERCISE}

For the whole group, left ventricular ejection fraction ranged from 16 to 40 per cent with a mean of 28 per cent, not significantly different from rest. EFCS ranged from 17 to 50 per cent with a mean of 35 per cent. All six patients who showed a rise in total left ventricular ejection fraction had an increased EFCS, and of five with a fall in total left ventricular ejection fraction, four had decreased EFCS. Only one patient had a decreased left ventricular ejection fraction with a rise in EFCS. Fig. 3 shows the changes in left ventricular ejection fraction on exercise plotted against the changes in EFCS, there being a significant correlation between the changes, whether positive or negative $(r=0.70$, $\mathrm{p}<0.01)$. Thus changes in ventricular function as assessed from the count-volume method reflect those from the geometric model using computergenerated ventricular perimeters.

Of seven with an increased stroke volume index on exercise, six had an increased EFCS. Of four

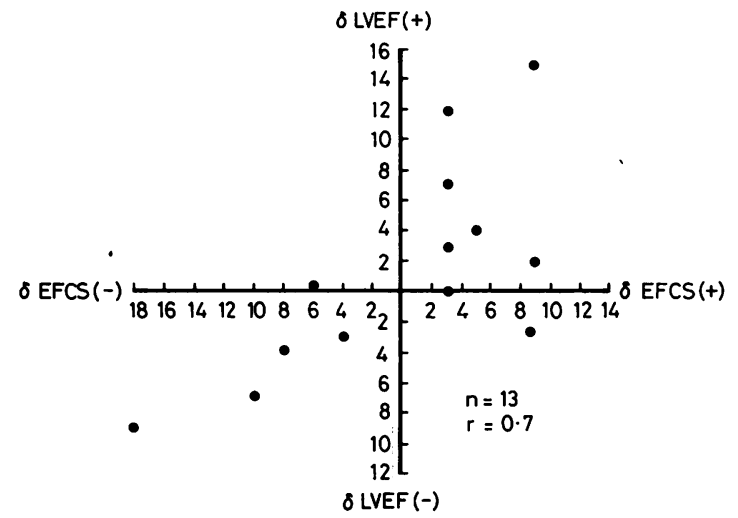

Fig. 3 Plot of change in left ventricular ejection fraction and change in EFCS from rest to submaximal exercise. $\delta L V E F$, change in total left ventricular ejection fraction (count volume method). $\delta E F C S$, change in ejection fraction of contractile segment.

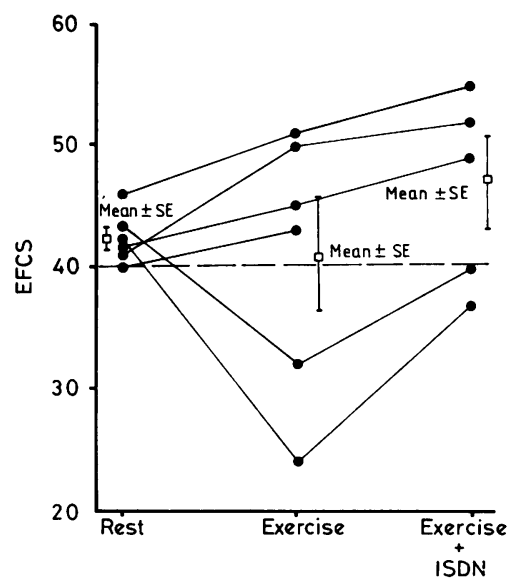

Fig. 4 Changes in EFCS in six patients with initial EFCS of 40 per cent and more.

with a reduced stroke volume index, three had reduced EFCS. The monotonic relation between EFCS and stroke volume index was maintained on exercise, as shown in Fig. 2b, with a correlation coefficient of 0.72 using Spearman's non-parametric rank test.

Of the six patients with an initial EFCS of 40 per cent or more, four showed an increase and two a decrease at submaximal exercise. Mean EFCS for this group did not differ significantly from the resting values (Fig. 4). Of those with an initial EFCS of less than 40 per cent, four improved and three deteriorated on exercise. Again, the mean value did not differ significantly from rest (Fig. 5).

\section{EFFECT OF CORONARY STATUS ON EXERCISE RESPONSE}

Of the eight with one-vessel disease, three improved and five deteriorated on exercise. All patients with more extensive disease improved. No patient experienced angina during exercise.

\section{EXERCISE AND ISOSORBIDE DINITRATE}

At exercise after isosorbide dinitrate, left ventricular ejection fraction ranged from 22 to 44 per cent with a mean of 32 per cent. This represents a significant improvement over the pre-isosorbide dinitrate values $(p<0.01$, Fig. 6). Stroke volume index ranged from 29 to $74 \mathrm{ml} / \mathrm{m}^{2}$, mean 41.4 , significantly better than before isosorbide dinitrate $(p<0.005)$.

EFCS ranged from 26 to 55 per cent with a mean of 38 per cent, eight patients showing an improvement, three being unchanged, and one worse than before isosorbide dinitrate, though for the whole group the improvement in EFCS failed to achieve statistical significance. 
Analysis of the responses in relation to the resting EFCS shows that of the five patients with resting EFCS of 40 per cent or more who received isosorbide dinitrate, all improved, with a mean EFCS for this subgroup of 46.6 compared with 40.9 before isosorbide dinitrate (Fig. 4). This improvement achieves statistical significance $(p<0.025)$. Of the seven with an EFCS of less than 40 per cent at rest, three improved after isosorbide dinitrate, one worsened, and three remained unchanged after isosorbide dinitrate (Fig. 5). All three who improved achieved a higher EFCS than at rest. Mean EFCS was 32.0 after isosorbide dinitrate, compared with 29.2 before the drug, an improvement that is not statistically significant.

Fig 7 relates the changes that occurred in the two exercise periods to the changes that occurred from rest to exercise. Of the seven patients in whom EFCS improved from rest to exercise, six improved further after isosorbide dinitrate and one worsened. Of the five who deteriorated from rest to exercise, two improved and three were unchanged after isosorbide dinitrate. Thus a bad response to exercise did not preclude a good response to isosorbide dinitrate, though a good response to exercise was more likely to predict a good response to isosorbide dinitrate.

Fig. 8 shows an example of how contractile segment function may improve after isosorbide dinitrate. The left-hand image shows the perimeters for the pre-isosorbide dinitrate study, the right shows them post-isosorbide dinitrate. The end-diastolic perimeter is shown in white, the endsystolic perimeter in yellow. It is evident that the

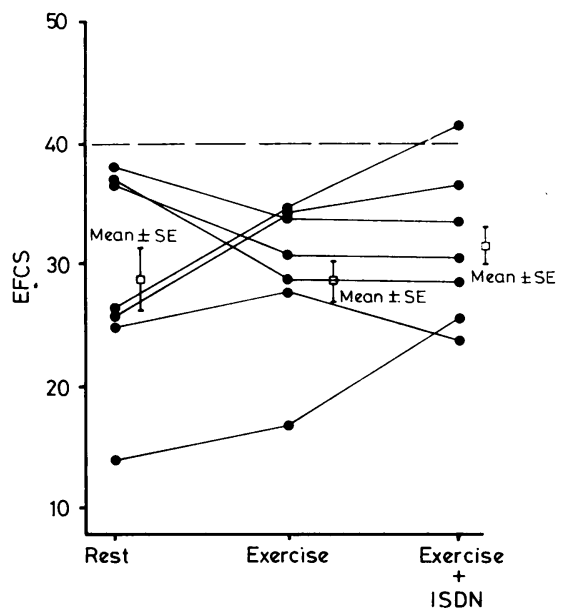

Fig. 5 Changes in EFCS in seven patients with initial EFCS of less than 40 per cent. EFCS, ejection fraction of contractile segment.

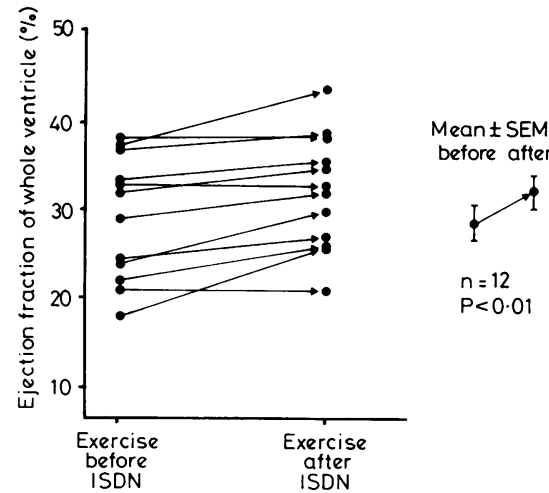

Fig. 6 Exercise left ventricular ejection fraction (LVEF) before and after isosorbide dinitrate (ISDN). SEM, standard error of the mean.

contractile segment shows greater inward motion in the right-hand image, and the calculated EFCS has correspondingly increased. The contractile segment and the aneurysmal segment are both clearly seen in each image.

EFFECT OF CORONARY STATUS ON ISOSORBIDE DINITRATE RESPONSE

Of the eight patients with one-vessel disease, five improved after isosorbide dinitrate and three remained unchanged. Of those who improved, three had shown an improvement from rest to exercise. Four of the five who improved had a resting EFCS of 40 per cent or more. Of those with three-vessel disease, three improved and one worsened. All had shown an improvement from rest to exercise and only one had a resting value of EFCS of 40 per cent or more.

These results suggest that the response to isosorbide dinitrate is not governed entirely by coronary status, inasmuch as the four non-responders contained three with one-vessel disease.

\section{Discussion}

Resection of left ventricular aneurysms is now a well-recognised surgical procedure, but it has become evident that not only is surgical mortality high in some series, ${ }^{45}$ but clinical improvement is by no means the rule. ${ }^{5}$ Key et al. ${ }^{16}$ commented on the necessity for 'good left ventricular deep constrictor activity' to increase the likelihood of a successful operation. In 1971, Kluge et al. ${ }^{17}$ described an unquantified relation between 'the quality of basal myocardial contractility' and immediate postoperative survival. More recently, these subjective relations have been superseded by 
objective methods for quantification of basal or contractile segment function from contrast angiograms. ${ }^{78}$

The importance of a method for objective quantification of contractile segment function is evident, as total left ventricular ejection fraction at rest was unable, in this study, to differentiate bad from good contractile segment function, an observation in agreement with Watson et al. ${ }^{8}$ All patients had reduced total left ventricular ejection fraction, however. The double hemispheroid model is easily applicable to the image produced by superimposition of the ventricular perimeters (Fig. 8) but is not the only method available. Arthur et al. ${ }^{7}$ calculated EFCS by excluding the aneurysmal portion of ventricle from the cineangiogram and redrawing the cardiac border as a fixed elliptical line equidistant from the longitudinal axis as the opposite contracting border. Lee et al. ${ }^{18}$ expressed the contractility of the nonaneurysmal ventricular segment as the difference between actual ejection fraction and that predicted from the spherical model of Feild et al. ${ }^{19}$ The double hemispheroid technique was chosen for this study as it has been validated against geometric models of known volumes, and the sums of subchamber volumes have been shown to correlate with total ventricular volumes. ${ }^{8}$

The relation between EFCS and stroke volume index (Fig. 2) is further testimony to the validity of the model as applied to the radionuclide study. Though others have reported no correlations between EFCS and cardiac index, ${ }^{8}$ this latter depends on another variable, heart rate, as well as on stroke volume.

The rationale behind separating those patients with an EFCS of 40 per cent or more from those

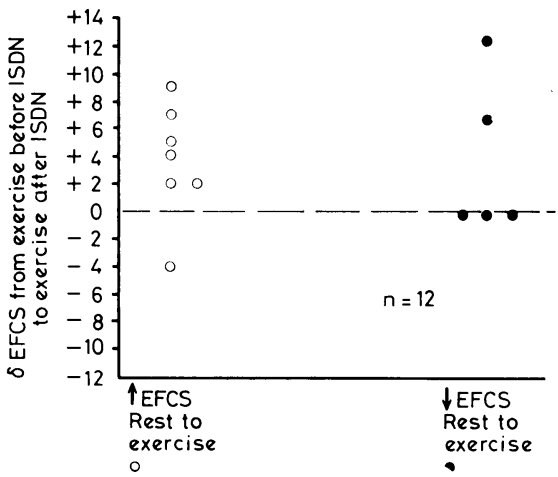

Fig. 7 Changes in EFCS from exercise before to after isosorbide dinitrate (ISDN) related to changes in EFCS from rest to exercise. EFCS, ejection fraction of contractile segment.

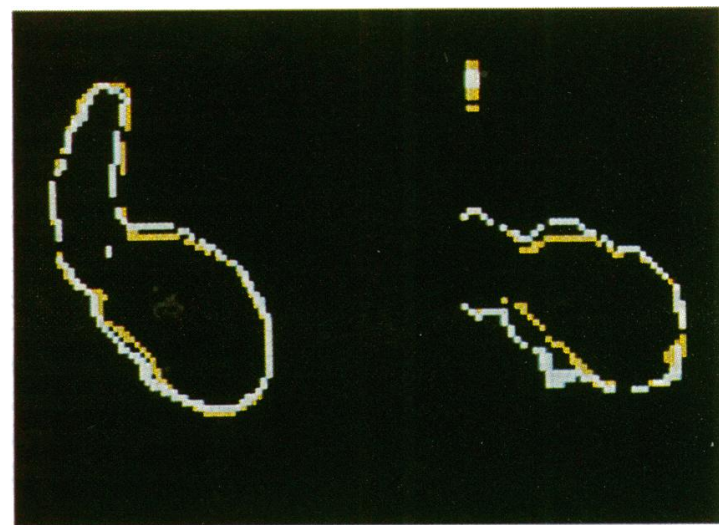

Fig. 8 Superimposed end-diastolic (white) and endsystolic (yellow) perimeters generated from the radionuclide ventriculogram by edge enhancement (see text). Left-hand image, exercise before isosorbide dinitrate (EFCS 24\%). Right-hand image, exercise after isosorbide dinitrate (EFCS improved to $36 \%$ ).

with a value of less than 40 per cent is based on the findings that an EFCS of less than 44 per cent in one study, ${ }^{8}$ or less than 38 per cent in another, ${ }^{7}$ predicted a poor surgical result. It is implied, therefore, that an EFCS of below approximately 40 per cent means a critical impairment of residual ventricular function.

The short data acquisition time required for a first pass radionuclide ventriculogram means that studies on exercise are not limited by changes in the stability of patients' circulatory status, all data collection being completed within one minute from the cessation of exercise.

Borer et al. ${ }^{20}$ have previously shown that no significant differences occurred in ejection fraction or regional ventricular function when sequential exercise radionuclide ventriculograms were performed with no intervention. This was the case for both normals and patients with coronary artery disease. The parallel changes between radionuclide and haemodynamic data described in this study support the findings of Borer et al. ${ }^{20}$ that changes seen after an intervention are not the result of intrinsic errors in the method used.

The changes in ventricular function, as measured by total left ventricular ejection fraction, were variable and were paralleled by changes in contractile segment function in 10 of the 13. The limitations of comparing results from a single plane geometric model with results from a three-dimensional measurement of radioactive counts may contribute to the discrepancies in the other three. It is possible that changes detected in the right anterior oblique may have been offset by opposing changes in the 
areas of the contractile segment not visualised in that projection, whereas this will not affect measurement of total left ventricular ejection fraction. The same problem will apply to comparisons of changes in EFCS and stroke volume index. The variable changes in left ventricular ejection fraction, EFCS, and stroke volume index that occurred on exercise are consistent with the spectrum of exercise responses described in other studies, in which wide ranges in the responses of stroke volume index, cardiac index, and left ventricular stroke work index occurred in patients with cardiac failure. ${ }^{21} 22$

Improvement in ventricular function on exercise may be brought about by augmented myocardial contractility under the inotropic stimulus of exercise and, indeed, the failing ventricle may be hypersensitive to circulating catecholamines. ${ }^{23}$ Conversely, the increase in peripheral vascular tone and hence ventricular afterload may be poorly tolerated by the failing ventricle because of the rise in systolic wall tension and myocardial oxygen consumption. ${ }^{24}$ Thus, the changes in EFCS, like the changes in stroke volume index, may be affected by multiple opposing factors and provide angiographic correlates of previously reported variable haemodynamic changes.

The failure of more than half the patients with single-vessel disease to improve EFCS on exercise, despite apparently normal residual coronary anatomy, is worthy of comment. It is possible that in the chronically dilated and overstretched ventricle excessive myofibrillar slippage leads to an often irreversible depression of contractility ${ }^{25}$ which is independent of coronary status.

Ischaemia of the contractile segment would not appear to play an important role in determining the response to exercise in view of the good response of those with extensive coronary disease. In addition, no patient experienced angina pectoris during exercise.

In recent years, dramatic improvements in the performance of the failing left ventricle have been shown after the administration of vasodilator drugs. Oral isosorbide dinitrate has been shown to be effective in causing a substantial reduction in left ventricular filling pressure in patients with congestive cardiac failure. ${ }^{26} 27$

The results of this study indicate that sublingual isosorbide dinitrate may lead to improvements in global and segmental ventricular function on exercise, with significant improvements in total left ventricular ejection fraction for the group as a whole, though the changes in EFCS were only statistically significant in those with a resting EFCS of greater than 40 per cent. In the other group, the changes in EFCS paralleled those in left ventricular ejection fraction and stroke volume index but did not achieve statistical significance. In four patients in the study (33\%) EFCS failed to improve, however.

The detected improvement in exercise EFCS after isosorbide is consistent with the improvement in exercise haemodynamics that has been previously reported in patients with both diffuse impairment of left ventricular function ${ }^{22}$ and with left ventricular aneurysms. ${ }^{28}$ In both these studies, isosorbide dinitrate caused significant improvements in ventricular stroke work index and stroke volume index, and significant reductions in left ventricular filling pressure on exercise.

It has been suggested that the principal action of vasodilators in general is to reduce left ventricular afterload $^{29}$ with a concomitant reduction in myocardial wall tension. The reduction in filling pressure may be also partly a result of a fall in preload produced by venodilatation. ${ }^{30}$ The reduced wall tension leads to an increased velocity of myocardial fibre shortening. ${ }^{31}$ In addition, the effects of the inotropic stimulation occurring on exercise may be enhanced by the pharmacological reduction of wall tension leading to greater augmentation of cardiac performance. ${ }^{32}$

It is of interest that the more consistent response to isosorbide dinitrate took place in the patients with the higher resting EFCS and the improvement was significant in this group but not in those with an EFCS of less than 40 per cent. A previous study has shown that the improvement in exercise cardiac index was related directly to the resting EFCS. ${ }^{22}$ Nevertheless, improvements in EFCS did occur in four out of seven patients with an EFCS of less than 40 per cent. It is also of note that responses to isosorbide dinitrate could not be entirely predicted on the basis of the rest to exercise changes, though of the eight who improved after isosorbide dinitrate, six had shown an improvement on exercise.

Again, coronary status did not appear to be of paramount importance in governing the response to isosorbide dinitrate, there being a similar spectrum of response in those with single or multiple vessel disease. The possibility does exist, however, of redistribution of blood by the nitrate to ischaemic areas in the contractile segment, ${ }^{33}$ though the evidence that such alterations in regional blood flow do occur in man is by no means conclusive.

Left ventricular aneurysmectomy may or may not lead to an improvement in exercise tolerance and left ventricular end-diastolic pressure ${ }^{5}$; so a method that could predict the likely results of surgical resection in terms of mortality and of clinical improvement would be welcome. 
One hopes that the pharmacological improvements in EFCS, left ventricular ejection fraction, and stroke volume index brought about by the vasodilator would mimic the beneficial haemodynamic effects of reducing ventricular filling pressure by aneurysmectomy, though further prospective and postoperative studies are required before a pharmacological intervention can be extrapolated to the surgical situation with any confidence. Contractile reserve, as measured by response to isosorbide dinitrate, does exist even in those patients with a low resting EFCS, though apparently to a lesser extent than in those with higher EFCS. Conversely, a resting EFCS of 40 per cent or more does not always indicate good contractile reserve, since some such patients will have had a bad response to exercise, and may not achieve resting levels even after isosorbide dinitrate. Even patients with one-vessel coronary disease may fall into this category possibly because of irreversibly damaged muscle in the contractile segment despite normal residual perfusion. It remains to be seen at what level of response, either good or bad, the surgical result becomes unacceptably poor.

In summary, computerised first pass radionuclide ventriculography provides a simple and safe way of assessing segmental and global ventricular function at rest and exercise. The double hemispheroid model is applicable to computer-generated ventricular outlines and detected changes in contractile segment function parallel changes in simultaneously recorded haemodynamics. If the function of the contractile segment is the prime factor in determining the result of aneurysmectomy, then stress radionuclide ventriculography can provide unique angiographic data to aid in selection of patients for aneurysmectomy.

The demonstration that isosorbide dinitrate can improve exercise ventricular function suggests that the drug may improve exercise tolerance in some patients with left ventricular aneurysms. This technique may allow more logical identification of patients likely to benefit from long-term nitrates when aneurysmectomy is not contemplated or contraindicated.

\section{References}

${ }^{1}$ Favoloro RG, Effler DB, Groves LK, Westcott RN, Suarez E, Lozada J. Ventricular aneurysm-clinical experience. Ann Thorac Surg 1968; 6: 227-45.

${ }^{2}$ Tice DA, Cheng TO, Dolgin M. Surgical treatment of postmyocardial infarction scars (ventricular aneurysms). Am Heart f 1970; 80: 282-6.

${ }^{3}$ Kouchoukos NT, Doty DB, Buettner LE, Kirklin JW. Treatment of postinfarction cardiac failure by myo- cardial excision and revascularization (abstract). Circulation 1971; 43/44 Suppl. II: 108.

${ }^{4}$ Graber JD, Oakley CM, Pickering BN, Goodwin JF, Raphael MJ, Steiner RE. Ventricular aneurysm: an appraisal of diagnosis and surgical treatment. Br Heart f 1972; 34: 830-8.

${ }^{5}$ Fisher VJ, Alvarez AJ, Shah A, Dolgin M, Tice DA. Left ventricular scars: clinical and haemodynamic results of excision. Br Heart $\mathcal{F} 1974$; 36: 132-8.

${ }^{6}$ Hamilton GW, Murray JA, Kennedy JW. Quantitative angiocardiography in ischemic heart disease. The spectrum of abnormal left ventricular function and the role of abnormally contracting segments. Circulation 1972; 45: 1065-80.

'Arthur A, Basta L, Kioschos M. Factors influencing prognosis in left ventricular aneurysmectomy (abstract). Circulation 1972; 45/46 Suppl. II : 127.

${ }^{8}$ Watson LE, Dickhaus DW, Martin RH. Left ventricular aneurysm: pre-operative hemodynamics, chamber volume and results of aneurysmectomy. Circulation 1975; 52: 868-73.

${ }^{9}$ Rigo P, Murray M, Strauss HW, Pitt B. Scintiphotographic evaluation of patients with suspected left ventricular aneurysm. Circulation 1974; 50: 98591.

${ }^{10}$ Dymond DS, Jarritt PH, Britton KE, Spurrell RAJ. Detection of post-infarction left ventricular aneurysms by first pass radionuclide ventriculography using a multicrystal gamma camera. Br Heart $f$ 1979; 41: 68-78.

${ }^{11}$ Mullins CB, Leshin SJ, Mierzwiak DS, Alsobrook $\mathrm{HD}$, Mitchell JH. Changes in left ventricular function produced by the injection of contrast media. Am Heart f 1972; 83: 373-81.

${ }^{12} \mathrm{Ganz}$ W, Swan HJC. Measurement of blood flow by thermodilution. Am f Cardiol 1972; 29: 241-6.

${ }^{13}$ Budinger TF, Rollo FD. Physics and instrumentation. Prog Cardiovasc Dis 1977; 20: 19-53.

${ }^{14}$ Kitamura S, Kay JH, Krohn BG, Magidson O, Dunne EF. Geometric and functional abnormalities of the left ventricle with a chronic localized noncontractile area. Am f Cardiol 1973; 31 : 701-7.

${ }^{15}$ Marshall RC, Berger HJ, Costin JC, et al. Assessment of cardiac performance with quantitative radionuclide angiography. Sequential left ventricular ejection fraction, normalized left ventricular ejection rate and regional wall motion. Circulation 1977; 56: 820-9.

${ }^{16} \mathrm{Key}$ JA, Aldridge HE, MacGregor DC. The selection of patients for resection of left ventricular aneurysms. f Thorac Cardiovasc Surg 1968; 56: 477-83.

${ }^{17}$ Kluge TH, Ullal SR, Hill JD, Kerth WJ, Gerbode F. Dyskinesia and aneurysm of the left ventricle. Surgical experience in 36 patients. $\mathcal{F}$ Cardiovasc Surg 1971; 12: 273-80.

${ }^{18}$ Lee, DCS, Johnson RA, Boucher CA, Wexler LF, McEnany MT. Angiographic predictors of survival following left ventricular aneurysmectomy. Circulation 1977; 55/56 Suppl. II : 12-18.

${ }^{19}$ Feild BL, Russell RO Jr, Dowling JT, Rackley CE. Regional left ventricular performance in the year following myocardial infarction. Circulation 1972; 46: 679-89. 
${ }^{20}$ Borer JS, Bacharach SL, Green MV, Kent KM, Johnston GS, Epstein SE. Effect of nitroglycerin on exercise-induced abnormalities of left ventricular regional function and ejection fraction in coronary artery disease. Assessment by radionuclide cineangiography in symptomatic and asymptomatic patients. Circulation 1978; 57: 314-20.

${ }^{21}$ Ross J Jr, Gault JH, Mason DT, Linhart JW, Braunwald E. Left ventricular performance during muscular exercise in patients with and without cardiac dysfuntion. Circulation 1966; 34: 597-608.

${ }^{22}$ Stephens JD, Camm AJ, Spurrell RAJ. Haemodynamic effects of isosorbide dinitrate in patients with congestive cardiac failure at rest and during submaximal supine exercise. Arch Mal Coeur 1978; 71: 241-50.

${ }^{23}$ Spann JE Jr, Buccino RA, Sonnenblick E, Braunwald E. Contractile state of cardiac muscle obtained from cats with experimentally produced ventricular hypertrophy and heart failure. Circ Res 1967; 21 : 341-54.

${ }^{24}$ Braunwald E. Control of myocardial oxygen consumption: physiological and clinical considerations. $\mathrm{Am} \mathcal{F}$ Cardiol 1971; 27: 416-32.

${ }^{25}$ Mason DT. Regulation of cardiac performance in clinical heart disease. Interactions between contractile state mechanical abnormalities and ventricular compensatory mechanisms. Am $\mathcal{f}$ Cardiol 1973; 32: 43748.

${ }^{26}$ Franciosa JA, Mikulic E, Cohn JN, Jose E, Fabie A. Hemodynamic effects of orally administered isosorbide dinitrate in patients with congestive cardiac failure. Circulation 1974; 50: 1020-4.
${ }^{27}$ Williams DO, Bommer WJ, Miller RR, Amsterdam EA, Mason DT. Hemodynamic assessment of oral peripheral vasodilator therapy in chronic congestive heart failure. Prolonged effectiveness of isosorbide dinitrate. Am f Cardiol 1977; 39: 84-90.

${ }^{28}$ Stephens JD, Dymond DS, Spurrell RAJ. Effects of afterload reduction in exercise left ventricular performance in patients with left ventricular aneurysm. Trans Eur Soc Cardiol 1978; 1 : 106.

${ }^{29} \mathrm{Cohn}$ JN. Vasodilator therapy for heart failure. The influence of impedance on left ventricular performance. Circulation 1973; 48: 5-8.

${ }^{30}$ Mason DT, Braunwald E. Effects of nitroglycerin and amyl nitrite on arteriolar and venous tone in the human forearm. Circulation 1965; 32: 755-66.

${ }^{31}$ Sonnenblick E, Parmley WW, Urschel CW. The contractile state of the heart as expressed by forcevelocity relations. Am $\mathcal{F}$ Cardiol $1969 ; 23$ : 488-503.

${ }^{32}$ Miller RR, Awan NA, Joye JA, et al. Combined dopamine and nitroprusside therapy in congestive heart failure. Greater augmentation of cardiac performance by addition of inotropic stimulation to afterload reduction. Circulation 1977; 55: 881-4.

${ }^{33}$ Horwitz LD, Gorlin R, Taylor WJ, Kemp HG. Effect of nitroglycerine on regional myocardial blood flow in coronary artery disease. $f$ Clin Invest 1971; 50: $1578-84$.

Requests for reprints to Dr D S Dymond, Department of Cardiology, St. Bartholomew's Hospital, London EC1A 7BE. 